\title{
Proper methodologies for psychological and sociological studies conducted via the Internet
}

\author{
CLAIRE M. HEWSON \\ University of Edinburgh, Edinburgh, Scotland \\ DIANNA LAURENT \\ Southeasterm Louisiana University, Hammond, Louisiana \\ and \\ CARL M. VOGEL \\ University of Stuttgart, Stuttgart, Germany
}

\begin{abstract}
This paper outlines specific methodologies for conducting research via computer networks. We discuss advantages of Internet experimentation over previous modes of telecommunicationfacilitated research and characterize features of studies that can benefit from Internet access and those which are unlikely to. We point out pitfalls and suggest a range of potential solutions in terms of specific practical techniques for managing the design, dissemination, and collection of Internet materials. We also discuss techniques for minimizing attrition and for adapting to recalcitrance presented by "hacker" vandalism.
\end{abstract}

The Internet and its sisters are qualitatively different from the other forms of electronic connectivity (telegraph, wireless radio, television, telephone) realized in this century and the last. The global computer-mediated connectivity supplied by the Internet is about as accessible as radio and television, but it is interactive in a way that radio, television, and even telephones are not. This paper outlines specific methodologies for conducting research via the Internet and suggests advantages over previous modes of telecommunication-facilitated research. We point out pitfalls and suggest a range of potential solutions.

The "Infobahn" links academic, government, military, commercial, and private interests primarily via networked computer systems. Academic links mobilize potential subject pools into categories roughly sorted by age and academic level (grammar and secondary schools have connections in addition to undergraduate and graduate institutes). Moreover, commercial Internet access providers are proliferating. A January 1996 survey (Lottor, 1996) indicates 9.472 million hosts, with 1.79 million in U.S. academic domains and 2.43 million in commercial domains. Private access servers like CompuServe are in the commercial domain and offer access internationally.

D.L. is in the Department of English at Southeastern Louisiana; C.M.V. is in the Institute for Computational Linguistics at Stuttgart. Correspondence concerning this article should be addressed to C. $M$. Hewson, Centre for Cognitive Science, University of Edinburgh, 2 Buccleuch Place, Edinburgh EH8 9LW, Scotland (e-mail: hewson@ cogsci.ed.ac.uk).
Other links supply a wider diversity of people, and this diversity expands every day. The increasing diversity is interesting for two reasons: it inarguably increases the availability of samples whose diversity approximates that of the entire population, and it also makes distinct groups directly accessible for research specific to the group definition. General connectivity among private users seems to select for people with a certain amount of interest in (or at least minimal fear of) technology. School and university connections abstract over that point because of interdisciplinary required use of network facilities, but segregate subjects more easily by age and personal/academic interest categories. Global connections along similar demographic lines allow attunement of research to language differences as well (important for psycholinguistic research). Note that the July 15, 1995, report on international connectivity (Landweber, 1995) indicates that there are 173 distinct "entities" (roughly meaning "nations") with widespread international access through BITNET, IP, or UUCP Internet links. This permits cross-cultural research on a scale never before possible. However, it is impossible to estimate the number of actual users, since most computers are in multiple-user environments.

We do not address here protocols for successful collaboration, nor methods for conducting research based on gleaning information from electronic archives. Rather, we consider the Internet as a mode of access to participants in experiments in scientific research and offer sound methodologies for using the Internet to collect data from questionnaires and software instruments for more directly interactive forms of experimentation. 


\section{ADVANTAGES OF INTERNET ACCESS}

It might seem that the Internet does not provide facilities for conducting psychological or sociological experiments beyond what is already available to experiments administered via telephone or post, because of the expressively limited modality in which the communication occurs. ${ }^{1}$ These media are generally cheaper and more practical to utilize than face-to-face administration of experiments. While it is generally assumed that face-toface administration yields higher response rates, it has also been shown that over time, face-to-face response rates decline, whereas mailed instruments, for example, remain free from decline (Goyder, 1985). Thus, though there may be claims of dehumanization (which researchers in human-computer interaction are striving to reduce), it is not obvious that face-to-face experimentation is the method of choice even in a financially ideal world. Telephone experimentation, as well as face-to-face interactions, have been acknowledged as susceptible to subjectexperimenter effects - that is, findings resulting from factors other than the one intended by the experimenter (Cotter, Cohen, \& Coulter, 1982). Various methods and techniques have been suggested to help reduce such effects, and Internet research seems especially to lend itself to these methods. One widely discussed artifact is the influence upon subjects of the demand characteristics of the experimental situation; for example, subjects may feel a need to please the experimenter or a pressure to conform to social norms. Suggested strategies for reducing these effects are particularly amenable to experimentation via the Internet. For example, by using the Internet, it is easy for subjects to respond anonymously; unlike in face-to-face interaction or phone interviews, subjects responding by anonymous e-mail can be secure that their identity is not known to the experimenter. ${ }^{2}$ There is evidence that guaranteeing anonymity and confidentiality of subjects' responses reduces the effects of factors due to demand characteristics and conformity to social norms (Esposito, Agard, \& Rosnow, 1984), thus encouraging cooperation and honesty from subjects.

Anonymity can also help reduce effects of the attributes and behavior of experimenters, these having been acknowledged as major factors in producing experimental demand characteristics. Experimenter anonymity serves to reduce possible effects of subjects responding differently to experimenters with different biosocial attributes (e.g., sex, age, race, and so on). Subject anonymity can help counter the opposite problem, that of experimenters responding differently to subjects with different biosocial attributes (see, e.g., R. Rosenthal, 1967). It has also been found that experimenters varying in biosocial attributes will behave differently toward their subjects and elicit different responses; male experimenters, for example, have been found to be more friendly toward their subjects than female experimenters are (R. Rosenthal, 1977). Anonymity of experimenters will not help reduce this effect, though it has been suggested that minimal contact between subject and experimenter is an ef- fective way of minimizing the problem; research carried out through the Internet is advantageous in this sense, because it is a more impersonal form of communication than face-to-face interaction or phone interaction, particularly when anonymity is preserved and contact deliberately minimized.

Mail surveys also allow a degree of anonymity, but they are less flexible in terms of allowing mutually anonymous follow-up and interactions between the experimenter and subjects. Also, low response rates have given researchers a reluctance to depend on that mode of experimentation (Dillman, Carpenter, Christenson, \& Brooks, 1974). In a context in which a representative percentage of the general population has Internet access, Internet administration of the same experiments is the cheapest and most effective solution to these problems. With some caveats detailed below, any instrument (questionnaire, survey, exam, etc.) that can be posted in the mail can also be transmitted across the Internet. Moreover, it is possible to nullify gender and race effects in this medium, while still maintaining fairly direct contact with the participants to provide a return rate that competes with face-toface experimentation. Internet experimentation also has a critical advantage over other interactive modes of experimentation because it can occur at the participant's leisure.

For an enormous variety of experimental questions, access to participants via the Internet can facilitate research whose analysis depends on statistical tests of extremely low power. Of course, there may be experimental questions that will be affected by the implied technological prowess of networked individuals. For example, tests of logical reasoning (Hewson \& Vogel, 1994) can be affected by the fact that a large (yet decreasing) percentage of the networked population consists of computer scientists who tend to have extensive training in symbolic logic. Nonetheless, the $>$ are ways to accommodate many of these issues by res icting the sources of participants. This is a workable solution because of the sheer volume of potential subjects. The approach that Hewson and Vogel (1994) used was to secure a sampling of students through a Canadian server for school students (SchoolNet), which they balanced with a sampling of older subjects identified through calls for participation from a random selection of USENETdiscussion news groups (henceforth, news). ${ }^{3}$ In separate empirical work in the elucidation of folk psychological concepts that had far less mathematical content, Hewson (1994) was able to use news groups alone.

\section{METHODS OF RESEARCH}

What styles of research communication are facilitated by the Internet? What methodologies are there for ensuring the generalizability of results and integrity of data? The new electronic connectivity is not necessarily a boon to researchers whose subject matter does not translate easily into ASCII characters and who lack more advanced facilities for viewing graphics easily. The first 
task is to identify research projects that could benefit from large-scale access to subjects and for which available software and technology make networked experimentation feasible. Surveys and questionnaires are perhaps the most obvious forms of research tool which lend themselves to administration via the Internet; these tools have previously been administered by means of face-toface interview, postal mail, or phone interaction. However, questionnaire research is not the only type that can be conducted through the Internet. For example, research on dialogue phenomena that emerge from cooperative tasks (cf. Garrod \& Anderson, 1987), as well as reaction time and even cross-modal experiments (cf. Swinney, 1979) are amenable to replication with networked subjects. However, hardware-rich experiments such as those required by eye-tracking research in psycholinguistics or face recognition are not enhanced by networked subjects for obvious reasons. Experiments whose data are to be studied by using statistical tests whose power demands a large subject pool are best facilitated by networked research, but experiments requiring fewer datapoints can also benefit-through comprehensive piloting, rapid replication, and multiple sampling.

We now briefly outline the sorts of research domains for which Internet access can be useful for experimentation. Guest log-in facilities like those provided by MUSEs (multi-user systems) and in which subjects interact inside virtual worlds or other programmed environments are obvious sources that could support tightly controlled experiments. The maze task experiments (Garrod \& Anderson, 1987; Garrod, Anderson, \& Sanford, 1984) are an example of research on semantic coordination, aministered over local area networks. In these experiments, subjects were confronted with identical mazes but different goal points and obstacles within the maze. The task was for pairs of subjects to guide each other through the mazes to the appropriate goal points while contending with the obstacles. This yielded a body of evidence about classes of conceptualization and coordination of conceptualizations. Advantages of generalization to Internet administration of the same experiment are obvious (see Healey, 1995). The questionnaire-oriented experiment of Hewson and Vogel (1994) was also successful in obtaining a large body of data pertaining to the AI literature on reasoning with natural language generics. However, not every subfield within the human sciences is as likely as others to gain from Internet access. Certainly, personality research, psychoanalysis, and learning can directly benefit. However, any benefit for intelligence testing is less obvious (notwithstanding the ETS objective to computerize its tests like the GRE), given the lack of control over access that the subject may have to external information sources (including other people). Research involving biofeedback is presently infeasible; study of nonverbal communication, virtually impossible. Within social psychology and sociology, group interactions, belief and attitude change, and stratification are all accessible. An advantage of the Internet over traditional modes of data collection in sociology derives from its flexibil- ity in combining the informal flexibility available to oral interviews with orders of magnitude of less expense (and therefore potential for a larger supply of interviews), and similarly, with questionnaires and questionnaire followups (Galtung, 1967). The essential characteristics of fields that can benefit from Internet-based experimentation are those in which written communication (immediate or reflected) suffices to supply data relevant to a problem at hand. Other forms of observation are possible, but considerably less feasible, given the current state of the Internet infrastructure.

\section{Materials}

Materials must be designed to facilitate simple transmission and readability in a variety of hardware environments. For instance, the participant pool is partitioned into subjects who have access only to ASCII terminals and line printers (and thus cannot process PostScript files either by previewing on bit-mapped screens or by printing the files on a PostScript laser printer) and those who have access to either or both advanced printing and previewing. ${ }^{4}$ In general, larger samples can be obtained by restricting materials to text files. This is, of course, because the average private user is bound by technological standards to be able to read 80 -column text files using the readable ASCII characters. This general restriction to ASCII characters does not preclude interactive or reaction time experiments; it does, however, restrict the sort of graphics that can be easily used within an experiment because of the equipment required in order to preview or print technologically advanced graphics. Graphics are not ruled out altogether from text files, but they are generally more laborious to produce, since the style of graphics is slightly more constrained than that which can be achieved by drawing a picture using solely the keystrokes of a basic typewriter. Research that hinges on the presentation of complex graphics is more constrained in its subject pools. Experimentation that involves users interacting with a program to create a data $\log$ is the other main genre of potential experimentation. Either genre is subject to the text/graphics partition, since it determines subject accessibility. Hewson and Vogel (1994) have presented research on data obtained via questionnaire in both classes of accessibility. The research question should determine whether questionnaire or interactive experimentation is best; it should be made clear, however, that interactive materials are still expensive to produce because there is not yet a large supply of reusable products and custom-built software is seldom cheap or portable.

\section{Modes of Access to Materials}

There are three stages to subject access of materials: call for participation, dissemination of materials, and collection of completed materials. Faced with seemingly arbitrary choices in modes of access, we emphasize maximal experimenter control over the materials. It turns out to be practical for the experimenter to create a computer account distinct from his/her personal research account 
on the computer system, mainly because of the volume of e-mail that such research can generate that is not directly related to the researcher's effective daily use of e-mail. It is also a convenient way to let the computer do some of the work of organizing, since separating the accounts by research project eliminates the problem of cross or misfiling of information. Of course it is always possible to set such separate accounts to forward mail to a personal account when an experiment is in a critical stage during which the experimenter should maintain close watch, and it is equally possible to give such a secondary account a personal rather than project-oriented user name to make the interactions with participants more human.

Call for participation. The initial step is best facilitated by random distribution among existing general discussion or disciplinary news groups and listservers. Random invitations should be prefaced with a brief indication of the researchers' credentials and seriousness of the research, in order to avoid provoking complaint about the appropriateness of the posting. In some cases, such as the Canadian SchoolNet project, a Gopher facility acts as a clearinghouse for ongoing proposals and projects involving student participation. To ensure a degree of commitment from participants, it is advisable for the initial invitation to include the researchers' contact addresses for negotiating actual administration of the materials, thus requiring more initiative from the subjects. This indirect method is distinguished from the direct method in which materials (e.g., surveys) are posted in the first step. The indirect method gives the experimenter more control over the investigation as a whole, since it is still possible to recover from faulty materials during piloting; if materials are broadcast directly from the outset, it will be impossible to follow up with revised materials, since it will be unknown what the effect of the initial broadcast will have been on potential subjects. Making an initial broadcast of an overview of the experiment with an invitation to participate leaves it up to the individual's initiative to respond to the experimenter. Giving the participant initiative is also a good way of dealing with and reducing attrition - for a start, it allows the experimenter to more correctly estimate the number of subjects who have looked at the materials, and thus makes it possible to calculate what the attrition rate actually is. Moreover, because participants have to take the initiative to volunteer to participate, it gives the experimenter greater faith that interest will be maintained through to the end of the experiment. We stress the indirect method here, because there already is a growing use of Internet news for surveys and questionnaires in which the materials are posted directly.

While M. Rosenthal and Rosnow (1975) have pointed out that the use of volunteer subjects can have a biasing effect on experimental results (e.g., they suggest that volunteers tend to be better educated than nonvolunteers, more sociable, and in higher need of social approval), they also outline several strategies that are effective in reducing such volunteers' biases. These strategies in- clude stating the theoretical and practical importance of the research, having the call for volunteers made by a person of as high status as possible, and making the appeal for volunteers sound as interesting and nonthreatening as possible. We recommend following these strategies, as far as possible, in order to improve generalizability of results by reducing any possible effects from volunteer bias.

Dissemination of materials. Administration of the materials to participants is most efficiently coordinated by e-mail, with the advantage of the natural paper trail created by incoming and outgoing mail logs built into most e-mail systems. A $\log$ of the various sorts of queries that emerge can be a valuable source of information in moving from pilot runs to actual experimentation. Materials themselves may also be administered via e-mail if an individual instance is not too lengthy (more than $100 \mathrm{~K}$ will overwhelm many mailers; if the document is compiled into PostScript by the researcher's word processing facilities, $100 \mathrm{~K}$ is about the length of a 6-pp. document). When the experiment does not involve interactive processing, and if the files that contain the materials are small enough, e-mail administration is the preferred method, because it affords absolute control over who obtains which experimental conditions. If files are too large for practical e-mail transfer, anonymous FTP (file transfer protocol) provides a useful alternative. Essentially, this is a program that a person can use to obtain guest access to networked host computers for the purposes of exchanging computer files. It is advisable, when making this use of FTP, to obtain a set of semantically unrelated directory names in which to store files pertaining to the various experimental conditions, without cross-reference to the other directories. The ideal is to locate the directories corresponding to different conditions at different (but shallow) positions in the system's FTP directory hierarchy. If the directories must remain at the same level in the FTP directory hierarchy, an even alphabetic distribution of their names is recommended as well. These precautions protect researchers from secondguessing "hackers" who might be inclined to poke around the system and "discover" materials that might prejudice their responses to assigned conditions. For interactive experiments, using FTP conditions will allow participants to obtain source programs that manage the interactive or executable versions of the programs which they can run directly in their hardware configuration without worrying about compilation on their systems. Ideal products in this line generate encrypted data files that the user need only e-mail back to the researcher. Internet accessibility allows the researcher to enter into protracted debriefing discussions after the experiment, the promise of which is an easy way to make participation in such research engaging to the subject in the first place.

Collection of completed materials. It is best to have completed materials returned via e-mail if it is a noninteractive study. An alternative would be to invert the dissemination procedure - for instance, to allow subjects to $u p$ load files to a designated space. However, controls against 
multiple uploads from a single person are difficult to maintain in an FTP interaction. Using e-mail thus offers greater control. An alternative is to adopt an interactive mode for both dissemination and return of materials.

Guest servers provide an important facility for making interactive participation possible for users who are not technically versed in compiling software or whose hardware is incompatible with any of the provided executables. Guest servers provide the additional security to the researcher of protecting source code from curious participants. In this mode, users log onto the researcher's host system as for any other multiuser system (MUDS/ MUSES) and are automatically engaged in the program that is designed to record activity. This mode of experimentation offers the greatest potential for future exploration, since it is possible to create "virtual worlds" in these interactive systems in which subjects meet with other subjects electronically and participate in competitive or collaborative tasks. Given recent psycholinguistic interest in dialogue corpora (cf. Anderson et al., 1992), we conjecture that these tools will see an explosion in experimental use in the short and medium term. Guest server facilities with data logs provide greater security to researchers than does providing copies of the interactive programs to users, which might leave the researcher dependent on the participant's promptly mailing back the completed materials. Interactive experiments of this nature are already starting to make an appearance on the Net, using the rudimentary interactive processing supplied by Mosaic - a public domain graphical user interface to a range of networked facilities such as FTP, GOPHER, ARCHIE, etc. (the sum of which are now known as the World-Wide Web). Heylighen and Bollen (1996) have recently announced an experiment in $\mathrm{HCI}$ investigating the adaptive organization of link structures in hypertext documents based on participants' link traversals. This mode of interface to experiments is potentially quite powerful, since it can be used to spawn the right kind of program on a user's machine to display graphics or whatever is appropriate to the experiment at hand, while the user provides feedback by clicking with a mouse or typing text into an appropriate window.

While e-mail administration of experiments is ideal for administering materials in the first place, interactive programs provided through access to the experimenter's host machine give the best guarantee that materials will be returned, since they can be collected on-line through session logs and the like. Programs run on the participant's home machine can, as has been said, be constructed to generate data files that the user need only mail back (and in some cases the program can mail the files directly to the experimenter from the user's account). It is slightly more difficult to guarantee the return of materials that the experimenter sends to the user directly; however, e-mail reminders are cheaper and much less obtrusive than follow-up visits or telephone calls. A real drawback that this method of experimentation does impose is a general loss of participants' scratch notes for calculating responses to some types of experimental items. Yet even this loss can be mitigated by declaring at the outset that subjects will be reimbursed for postage required to mail materials to the experimenter or by encouraging the subject to construct or translate materials into electronic form to be e-mailed back as a companion to the completed materials.

Guarding against recalcitrance. One potential hindrance to research is the variety of vandalism in computer systems referred to as "hacking." Networked experimentation does make itself liable to this; however, there are some quite easy protections that can secure researchers from casual hackers. These solutions include write protecting files put out for FTP, and resisting the acceptance of "patches" offered by participants to correct "bugs" in source code left for subjects to FTP. Easier still is to take care to use e-mail administration of the materials so that who has participated in which experiment is recorded. That is, the call for participation should not direct the reader immediately to the FTP'location where materials are available, but instead direct the reader to contact the experimenter to be notified of the relevant information.

Occasional electronic harassment in response to the fact that a call for participation has been cross-posted to randomly selected news groups is a recent problem, but it has no real impact on the validity or reliability of the findings, although it can affect administration. When one posts the initial call for participation to random news groups, it is important that one use cross-posting to multiple groups rather than multiple individual postings. This saves disk space on computer systems that subscribe to those news groups, and it also saves considerable hassle and harassment from network vigilantes who monitor news groups for multiply posted messages. Nonetheless, even random cross-postings that detail research credentials and proffer a preemptive apology can still elicit e-mail complaints from irritated news readers. It is advisable to keep copies of all related correspondence, for such phenomena will surely soon be of interest to social psychologists and researchers who study interpersonal communication! This and other forms of potential harassment are additional motivations for administering networked research using separate accounts for each project to prevent such correspondence from affecting the researcher's personal mailbox.

To recapitulate, the integrity of materials is best preserved when the researcher maintains maximal control, with a minimum of transactions in which materials are exchanged.

In this paper we have tried to introduce the Internet as a point of access to participants in experiments in the human sciences. We have outlined a sound methodology for conducting research on the net; this methodology has been developed in our own research, and we encourage others also to use it. 


\section{REFERENCES}

Anderson, A. H., Bader, M., Bard, E. G., Boyle, E. H., Doherty, G. M., Garrod, S. C., ISARd, S. D., KowtKo, J. C., MCAllister, J. M., Miller, J., Sotillo, C. F., Thompson, H. S., \& Weinert, R. (1992). The HCRC Map Task Corpus. Language \& Speech, 34, 351-366.

Cotter, P. R., Cohen, J., \& Coulter, P. (1982). Race-of-interviewer effects in telephone surveys. Public Opinion Quarterly, 46, 278-284.

Dillman, D. A., Carpenter, E., Christenson, J., \& Brooks, R. (1974). Increasing mail questionnaire response: A four state comparison. American Sociological Review, 39, 744-745.

Esposito, J., AGard, E., \& Rosnow, R. (1984). Can confidentiality of data pay off? Personality \& Individual Differences, 5, 477-480.

Galtung, J. (1967). Theory and methods of social research. New York: Allen \& Unwin.

Garrod, S. C., \& ANDERSon, A. (1987). Saying what you mean in dialogue: A study in conceptual and semantic co-ordination. Cognition, 27, 181-218.

Garrod, S. C., ANDERSon, A., \& SANFord, A. (1984). Semantic negotiation and the dynamics of conversational meaning (Tech. Rep. No. 1). University of Glasgow, Department of Psychology.

GOYDER, J. (1985). Face-to-face interviews and malled questionnaires: The net difference in response rate. Public Opinion Quarterly, 49, 234-252.

HEALEY, P. (1995). Communication as a special case of misunderstanding: Semantic coordination in dialogue. Unpublished doctoral dissertation, University of Edinburgh, Centre for Cognitive Science.

Hewson, C. (1994). Empirical evidence regarding the folk psychological concept of belief. In A. Ram \& K. Eiselt (Eds.), Proceedings of the Sixteenth Annual Conference of the Cognitive Science Society (pp. 403-406). Hillsdale, NJ: Erlbaum.

Hewson, C., \& Vogel, C. (1994). Psychological evidence for assumptions of path-based inheritance reasoning. In A. Ram \& K. Eiselt (Eds.), Proceedings of the Sixteenth Annual Conference of the Cognitive Science Society (pp. 409-414). Hillsdale, NJ: Erlbaum.

HEylighen, F., \& Bol.Len, J. (1996). Development and publication of systems knowledge on the Internet: The Principia Cybernetica Web. Available: http://pespmcl.vub.ac.be/papers/PapersFH.html Manuscript submitted for publication.
LANDWEBER, L. (1996). International connectivity: Version 14 [Online]. Madison: University of Wisconsin, Department of Computer Science. Available: ftp://ftp.cs.wisc.edu.

LoTTOR, M. (1996). Internet domain survey: January 1996 [On-line]. Available: http://www.nw.com or ftp://ftp.nw.com.

Rosenthal, M., \& Rosnow, R. (1975). The volunteer subject. New York: Wiley.

Rosenthal, R. (1967). Covert communication in the psychological experiment. Psychological Bulletin, 67, 356-367.

Rosenthal, R. (1977). Biasing effects of experimenters. ETC: A Review of General Semantics, 34, 253-264.

SwinNEY, D. A. (1979). Lexical access during sentence comprehension: $(\mathrm{Re})$ consideration of context effects. Journal of Verbal Learning \& Verbal Behaviour, 18, 645-59.

\section{NOTES}

1. In this paper we adopt a sense of "experiment" which encompasses forms of data collection beyond those involving the manipulation of tightly controlled variables; thus we refer to surveys and questionnaires, for example, as forms of experimentation.

2. E-mail can be arranged to be simply impersonal, giving no indication of identity, and it can be properly anonymous, even doubleblind, if one uses one of the extant anonymous e-mail servers (e.g., anon.penet.fi).

3. BITNET discussion lists are also possible; however, news offers considerably more flexibility in terms of moderation and administration (there need not be a moderator or list owner, for example). Readers do not need to know of the existence of a news group before stumbling into its discussions and have to undertake virtually no initiative to either join or exit.

4. Many computer novices access the network via Macintosh bitmapped video display units capable of previewing graphic materials prepared as PostScript files (or have access to PostScript laser printers).

(Manuscript received December 1, 1995; revision accepted for publication January 30,1996 .) 